\title{
Efectos potenciales del marisqueo sobre moluscos gasterópodos de interés comercial (Osilinus spp. y Patella spp.) en el Archipiélago Canario
}

\author{
Potential effects of man harvesting on gastropod molluscs of commercial interest \\ (Osilinus spp. and Patella spp.) in the Canarian Archipelago
}

\author{
Rubén Ramírez ${ }^{1}$, Fernando Tuya ${ }^{2,3}$ y Ricardo J. Haroun ${ }^{1}$ \\ ${ }^{1}$ Centro de Investigación en Biodiversidad y Gestión Ambiental (BIOGES, Universidad de Las Palmas de Gran Canaria, \\ Campus de Tafira s/n CP: 35017, Las Palmas de Gran Canaria, Islas Canarias, España \\ ${ }^{2}$ CIIMAR, Rua dos Bragas 289, 450-123 Porto, Portugal \\ ${ }^{3}$ Centre for Ecosystem Management, Edith Cowan University, 100 Joondalup Drive, \\ Joondalup, 6027 Western Australia, Australia \\ ftuya@yahoo.es
}

\begin{abstract}
This study assessed the effects of human influences over the abundance and size patterns of five species of gastropods (top-shell snails and limpets) commonly collected in the Canarian Archipelago: 'burgado hembra' (Osilinus atrata), 'burgado macho' (Osilinus sauciatus), 'lapa blanca' (Patella aspera), 'lapa negra' (Patella candei crenata) and 'lapa de sol' (Patella rustica). We studied patterns of abundance and size of these species across three islands (Lanzarote, La Graciosa and Alegranza) corresponding to three levels of human influence: high, medium and low, respectively; which were quantified through three indicators: human pressure (inhabitants $\mathrm{km}^{-1} \mathrm{~d}^{-1}$ ), accessibility to the coast ( $\mathrm{km}$ of sealed and unsealed roads), and volumes of capture (kg). The abundances of $O$. atrata and $P$. aspera were statistically higher at Alegranza than at La Graciosa and Lanzarote, whereas the density was 10 to 15 times larger at Alegranza for four of the five studied species. Significant differences in the size structure of the species among islands (= levels of human influence) were also observed. Not only all large-sized individuals disappeared at Lanzarote and La Graciosa, yet there was a decrease in numbers for the majority of size ranges. Thought populations of top-shell snails and limpets were affected by natural variability, human activities turned out to be the major driver for the observed differences among islands. These results seriously question the effectiveness of the current shellfish regulations contained in the Regional Fish Law.
\end{abstract}

Key words: intertidal, marine resources, overexploitation, Canary Islands

\section{Introducción}

En las últimas décadas, la mayor accesibilidad a las zonas costeras las hace susceptibles a una amplia variedad de impactos humanos (Thompson et al. 2002). Entre estos
Resumen.- Este estudio determinó los efectos de la influencia humana sobre la abundancia y la estructura de talla de cinco especies de moluscos gasterópodos comúnmente recolectados en el Archipiélago Canario: el 'burgado hembra' (Osilinus atrata), el 'burgado macho' (Osilinus sauciatus), la 'lapa blanca' (Patella aspera), la 'lapa negra' (Patella candei crenata) y la 'lapa de sol' (Patella rustica). Se analizaron tres islas (Lanzarote, La Graciosa y Alegranza) correspondientes a tres categorías de influencia humana: alta, media y baja, respectivamente; cuantificadas a través de tres indicadores: la presión humana (hab $\left.\mathrm{km}^{-1} \mathrm{~d}^{-1}\right)$, la accesibilidad a la costa $(\mathrm{km}$ de red viaria) y los volúmenes de extracción de los recursos (kg). La abundancia de O. atrata y $P$. aspera fue estadísticamente mayor en Alegranza que en La Graciosa y Lanzarote, mientras que los valores de densidad fueron de 10 a 15 veces superiores en Alegranza para cuatro de las cinco especies estudiadas. Para la estructura de talla, se detectaron notables diferencias entre islas (= niveles de influencia humana). En Lanzarote y La Graciosa no sólo desaparecieron los ejemplares de mayor talla, sino que disminuyeron los ejemplares para la mayoría de rangos de talla. A pesar que la variabilidad natural de las poblaciones de burgados y lapas es alta, la actividad humana parece ser la causa principal de las diferencias observadas entre islas. Estos resultados cuestionan seriamente la efectividad de las normas sobre marisqueo de la actual Ley de Pesca de Canarias.

Palabras clave: intermareal, recursos marinos, sobreexplotación, Islas Canarias

impactos destaca la extracción de organismos (e.g. para alimento, cebos o carnadas, muestras para acuarios, conchas para decoración), que provoca alteraciones, tanto de tipo directo como indirecto, sobre las poblaciones de la zona intermareal (Kingsford et al. 1991, Addessi 1994, 
Lindberg et al. 1998). Así, se producen principalmente cambios en la densidad y variaciones en la estructura de tallas de las poblaciones explotadas (Hockey \& Bosman 1986, Keough et al. 1993, Adessi 1994, Lindberg et al. 1998, Mannino \& Thomas 2001, Kido \& Murray 2003, Sagarin et al. 2007). De hecho, los mariscadores recolectan los ejemplares más grandes, ya que son más atractivos y aparentes (Hockey \& Bosman 1986, Cantos et al. 1994, Lindberg et al. 1998, Kido \& Murray 2003, Roy et al. 2003), además de presentar un mayor valor comercial. Como consecuencia, el éxito reproductor de tales especies puede verse mermado, provocando un descenso de las poblaciones y, en último lugar, posibles fenómenos de extinción (Nuñez et al. 2003, GuerraGarcía et al. 2004).

Los recursos marisqueros han sido aprovechados en numerosas regiones costeras del planeta (Hayward \& Brook 1981, Hockey \& Bosman 1986, Mannino \& Thomas 2001, Roy et al. 2003). Por ejemplo, las costas rocosas de la región macaronésica (Azores, Madeira, Islas Canarias e Islas de Cabo Verde) han sido explotadas desde épocas prehistóricas por los primeros pobladores prehispánicos (Hawkins et al. 2000, Batista 2001, Ferraz et al. 2001). Los organismos recolectados constituían un suplemento de comida (fuente de proteínas) o eran utilizados como moneda de cambio con otros productos (Batista 2001, Ferraz et al. 2001, Cabrera \& Cabrera 2004). Desde la conquista de las islas (siglo XV) hasta la época actual, esta actividad se ha seguido practicando a nivel recreativo, pero también a nivel profesional; es decir, los organismos capturados son vendidos para obtener un beneficio económico (Ferraz et al. 2001, Cabrera \& Cabrera 2004, Navarro et al. 2005). La existencia de multitud de concheros repartidos por todas las islas confirma esta explotación a lo largo del tiempo (Batista 2001). Sin embargo, la recolección de recursos marisqueros es mayor en la actualidad que en épocas pasadas, debido tanto al incremento de personas que los consumen como aquellas que los recolectan para su venta. Así, las poblaciones de algunos organismos (e.g. lapas) de la zona costera han disminuido notablemente su abundancia (Navarro et al. 2005, Ramírez et al. 2005) y, en casos extremos (e.g. Patella candei Orbigny, 1840), han llegado al borde de la extinción (Núñez et al. 2003).

Aunque algunos trabajos han descrito en qué estado de conservación se encuentran las poblaciones de determinados recursos marisqueros en el Archipiélago Canario (e.g. Núñez et al. 1994), existen escasos estudios que cuantifiquen con un diseño experimental adecuado cuáles son los efectos de la acción humana (Núñez et al. 2003, Navarro et al. 2005, Ramírez et al. 2005). El objetivo de este estudio fue, por tanto, determinar el efecto de la influencia humana sobre las poblaciones de ciertos recursos marisqueros de las costas rocosas del Archipiélago Canario. Específicamente, estudiamos los cambios en la abundancia y estructura de talla de cinco moluscos gasterópodos en áreas sujetas a diferente influencia humana, cuantificada no sólo a través de la explotación directa de los recursos marisqueros, sino utilizando otros indicadores relacionados con ésta, como la presión (o densidad) y accesibilidad poblacional sobre la costa. Suponemos que en áreas sujetas a una alta influencia humana, las especies presentan alteraciones poblacionales (e.g. disminución de abundancia y talla) respecto a áreas sometidas a menor influencia.

\section{Material y métodos}

\section{Área de estudio y diseño muestral}

Este trabajo se llevó a cabo en la isla de Lanzarote (L) y en los islotes adyacentes de La Graciosa (LG) y Alegranza (AG); los últimos están situados en la zona geográfica denominada 'Archipiélago Chinijo' (Fig. 1). En cada isla se seleccionaron dos localidades de $10 \mathrm{~s}$ de $\mathrm{m}$ de extensión, separadas desde 1 hasta 10s de km. Todas las localidades estaban constituidas por plataformas rocosas basálticas (origen volcánico) expuestas directamente al oleaje, que dominaba en frecuencia desde el norte (Tabla 1). Los muestreos se realizaron en la zona intermareal entre septiembre y octubre de 2004, aprovechando el régimen máximo de mareas durante esta época del año (0-3 m). En general, en la zona intermareal canaria se pueden diferenciar tres zonas (alta, media y baja) según los esquemas de zonación clásicos (Stephenson \& Stephenson 1949). La zona intermareal alta se caracteriza por la presencia de algas verde-azules (cianobacterias) como Calothrix crustacea; la zona media está dominada por el crustáceo cirrípedo Chthamalus stellatus; y la zona baja está cubierta por céspedes de macroalgas, principalmente algas rojas y pardas (Ramírez et al. 2008).

El diseño muestral incluyó, por tanto, tres islas (L, LG y AG) correspondientes a tres categorías de influencia humana: alta, media y baja, respectivamente (Tabla 2). Para cuantificar dicha influencia humana se utilizaron los siguientes indicadores: (1) presión humana sobre la costa, la cual se refirió al número de habitantes por km de perímetro costero y día (para su cálculo se usaron tanto el número de habitantes locales como los turistas foráneos); (2) accesibilidad a las zonas costeras, la cual se refirió $\mathrm{al} \mathrm{n}^{\circ}$ de $\mathrm{km}$ de carreteras y caminos accesibles a los vehículos; y (3) explotación marisquera, que se refirió a los volúmenes $(\mathrm{kg})$ de captura realizados por mariscadores profesionales (sólo se dispusieron de volúmenes totales para lapas, Patella spp., en el año 2008). 
Tabla 1

Características de las localidades estudiadas. *: sin población

Characteristics of the studied localities. *: without population

\begin{tabular}{|c|c|c|c|c|c|}
\hline Isla & Localidad & Extensión (m) & Orientación & Inclinación & $\begin{array}{l}\text { Distancia a población } \\
(\mathrm{km}) / \text { acceso rodado }(\mathrm{m})\end{array}$ \\
\hline \multirow[t]{2}{*}{ Lanzarote } & Las Caletas & 54 & SE $163^{\circ}$ & $<45^{\circ}$ & $0 / 0$ \\
\hline & El Cochino & 93 & NW $310^{\circ}$ & $<45^{\circ}$ & $7 / 5000$ \\
\hline \multirow[t]{2}{*}{ La Graciosa } & Caleta de Arriba & 60 & $\mathrm{SE} 140^{\circ}$ & $<45^{\circ}$ & $3 / 0$ \\
\hline & El Corral & 89 & NW $330^{\circ}$ & $<45^{\circ}$ & $5 / 0$ \\
\hline \multirow[t]{2}{*}{ Alegranza } & El Veril & 40 & SE $150^{\circ}$ & $<45^{\circ}$ & $* / 0$ \\
\hline & La Rapadura & 36 & $\mathrm{NE} 80^{\circ}$ & $<45^{\circ}$ & $* / 0$ \\
\hline
\end{tabular}

A

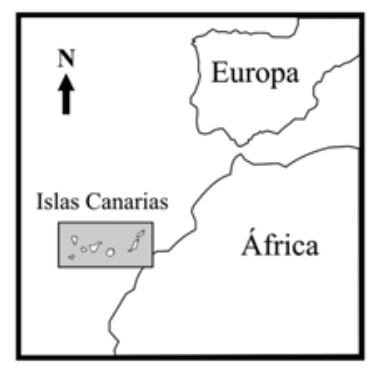

\section{B}

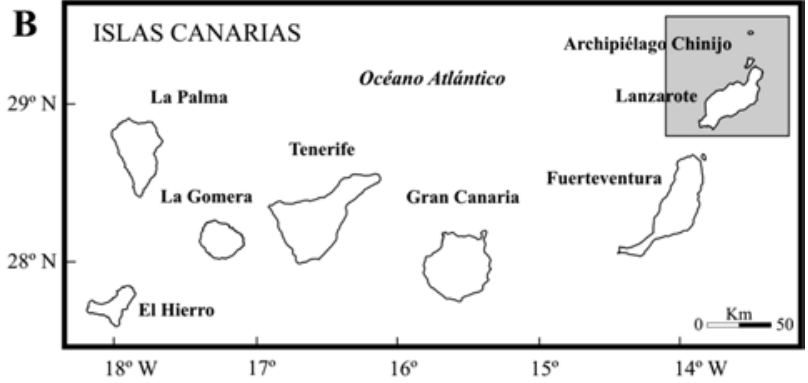

D

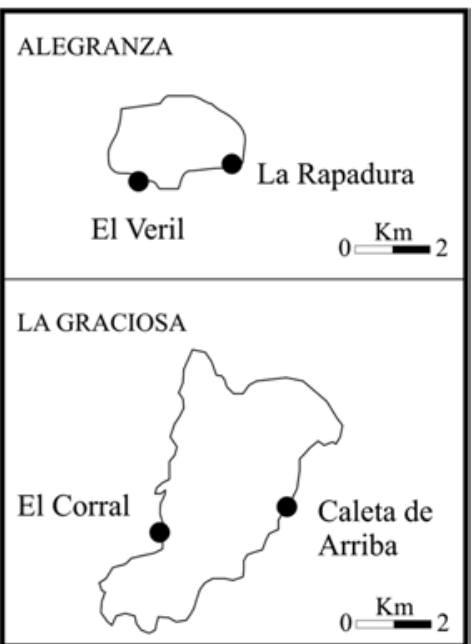

$14^{\circ} \mathrm{W}$

Figura 1

Área de estudio. A: Posición del Archipiélago Canario respecto del continente africano y europeo. B: Posición de la zona de estudio en el extremo oriental del Archipiélago. C: Posición de las localidades de muestreo (•) en Lanzarote,

La Graciosa y Alegranza (el cuadrado gris indica el área de reserva marina). D: detalle de las localidades muestreadas en Alegranza y La Graciosa

Study area. A: Location of the Canarian Archipelago relative to Africa and Europe. B: Location of the study area in the easternmost tip of the Canarian Archipelago. C: Location of sampled locations $(\bullet)$ at Lanzarote, La Graciosa and Alegranza (the grey box

denotes the area within the marine protected area). D: Detail of the sampled locations at Alegranza and La Graciosa 
Tabla 2

Estimación de los indicadores de influencia humana para las tres islas estudiadas

Estimated indicators of human influence at each studied island

\begin{tabular}{|c|c|c|c|c|c|c|}
\hline Isla/Influencia humana & $\begin{array}{l}\text { Km de } \\
\text { costa }\end{array}$ & $\begin{array}{c}\mathrm{N}^{\mathrm{o}} \text { de } \\
\text { habitantes } \\
(2004)\end{array}$ & $\begin{array}{l}\mathrm{N}^{\mathrm{o}} \mathrm{de} \\
\text { turistas } \\
(2004)\end{array}$ & Hab. $\mathrm{Km}^{-1} \mathrm{~d}^{-1}$ & $\begin{array}{c}\text { Km de red } \\
\text { viaria }\end{array}$ & $\begin{array}{c}\text { Volumen }(\mathrm{Kg}) \\
\text { de capturas } \\
(2008)\end{array}$ \\
\hline Lanzarote/Alta & 213,2 & 116.7 & 1.770 .176 & 569,4 & 457 & 675,6 \\
\hline La Graciosa/Media & 28 & 600 & 10.000 & 22,4 & 25 & 525 \\
\hline Alegranza/Baja & 14 & 0 & 0 & 0 & 0 & 0 \\
\hline
\end{tabular}

\section{Especies estudiadas y método de muestreo}

Se consideraron cinco especies de moluscos gasterópodos con interés marisquero, dos especies de tróquidos: el 'burgado hembra' Osilinus atrata (Wood, 1823) y 'burgado macho' Osilinus sauciatus (Koch, 1845) y tres especies de lapas: la 'lapa blanca' Patella aspera (Röding, 1798), la 'lapa negra' Patella candei crenata (Orbigny, 1840) y la 'lapa curvina' Patella rustica (Linnaeus, 1758).

La abundancia de los organismos se cuantificó mediante el uso de unidades de muestreo (cuadrados de $50 \times 50 \mathrm{~cm} ; 0,25 \mathrm{~m}^{2}$ ) distribuidas al azar sobre el sustrato, siguiendo así el método empleado previamente para el conjunto del Archipiélago Canario (Navarro et al. 2005, Ramírez et al. 2005), que también ha sido utilizado y reconocido como eficaz por otros autores para este tipo de trabajos (e.g. Cantos et al. 1994, Milazzo \& RamosEsplá 2000, Kido \& Murray 2003). En cada localidad, se colocaron al azar $n=90$ cuadrados, separados desde 1 hasta $10 \mathrm{~s}$ de $\mathrm{m}$, distribuyéndose en las tres zonas intermareales (alta, media y baja) de manera equitativa $(\mathrm{n}=30$ por zona). Dentro de cada cuadrado se contaron y midieron (precisión hasta $\mathrm{mm}$ ) mediante el uso de calibradores Vernier todos los individuos. Se midió la distancia desde el ápice hasta la base de la columbela para las especies de burgados (Osilinus spp.) y la longitud total de la apertura de la concha para las tres especies de lapas (Patella spp.).

\section{Análisis de datos}

Para contrastar las diferencias en abundancia entre islas, y por tanto entre categorías de influencia humana, para cada una de las especies consideradas, se ejecutaron ANOVAs, que incluyeron los factores: (1) isla (factor fijo, con tres niveles) y (2) localidad (factor aleatorio y anidado en el factor isla, con dos niveles). En aquellos casos donde la homogeneidad de las varianzas (prueba de Cochran) no se verificó a pesar de la trasformación de los datos, procedimos a fijar un nivel de confianza $(\alpha)$ más pequeño, y por tanto más conservador (Underwood 1997). Las diferencias entre islas se determinaron, en aquellos casos apropiados, mediante contrastes $a$ posteriori con una prueba SNK.

Para definir la estructura poblacional de cada especie se obtuvo un diagrama de tallas para la totalidad de los individuos de cada isla. Los rangos de talla se establecieron en intervalos de $3 \mathrm{~mm}$ para las especies de burgados y de $5 \mathrm{~mm}$ para las de lapas (Navarro et al. 2005, Ramírez et al. 2005). Para contrastar las diferencias en las distribuciones de frecuencias de tallas entre islas, es decir categorías de influencia humana, se emplearon tablas de contingencia, que contrastaron las diferencias a través del estadístico $\chi^{2}$.

\section{Resultados}

\section{Burgados (Osilinus spp.)}

Se registró un total de 835 ejemplares de $O$. atrata para todo el estudio, de los cuales aproximadamente un 74\% se encontró en AG, un $21 \%$ en LG y un $5 \%$ en L. Así, se detectaron diferencias significativas para la abundancia entre islas (Tabla 3); la abundancia fue superior en la isla de AG respecto a $\mathrm{LG}$ y $\mathrm{L}$ (prueba SNK, $0,01<P<$ 0,05; Fig. 2), que no mostraron diferencias significativas entre ellas. Por otro lado, se encontraron 82 individuos de $O$. sauciatus repartidos equitativamente entre L (50\%) y LG (50\%); no se encontró ningún ejemplar en AG. Sin embargo, no se detectaron diferencias significativas entre islas, pero sí entre localidades tanto para LG (prueba SNK, $0,01<P<0,05$ ) como para L (prueba SNK, $P<$ 0,01) (Tabla 3, Fig. 2). 
Tabla 3

Resultados del ANOVA contrastando las diferencias de abundancia para Osilinus atrata, Osilinus sauciatus, Patella aspera, Patella candei crenata y Patella rustica entre islas y localidades

Results of ANOVA testing for differences in abundances of Osilinus atrata, Osilinus sauciatus, Patella aspera, Patella candei crenata and Patella rustica among islands and locations

\begin{tabular}{|c|c|c|c|c|c|c|c|c|c|c|c|}
\hline \multirow[b]{2}{*}{ Fuente de variación } & \multirow[b]{2}{*}{ gl } & \multicolumn{2}{|c|}{ O. atrata } & \multicolumn{2}{|c|}{ O. sauciatus } & \multicolumn{2}{|c|}{ P. aspera } & \multicolumn{2}{|c|}{ P. candei crenata } & \multicolumn{2}{|c|}{ P. rustica } \\
\hline & & $\mathrm{Cm}$ & $\mathrm{F}$ & $\mathrm{Cm}$ & $\mathrm{F}$ & $\mathrm{Cm}$ & $\mathrm{F}$ & $\mathrm{Cm}$ & $\mathrm{F}$ & $\mathrm{Cm}$ & $\mathrm{F}$ \\
\hline Prueba de Cochran & & \multicolumn{2}{|c|}{$\mathrm{C}=0,51 * *$} & \multicolumn{2}{|c|}{$\mathrm{C}=0,57 * *$} & \multicolumn{2}{|c|}{$\mathrm{C}=0,68^{*} *$} & \multicolumn{2}{|c|}{$\mathrm{C}=0,75^{*} *$} & \multicolumn{2}{|c|}{$\mathrm{C}=0,57 * *$} \\
\hline Isla $=$ Is & 2 & 8540,11 & $9,72 *$ & 48,62 & 0,80 & 1127,02 & $32,88^{* *}$ & 928,35 & 5,15 & 475,05 & 3,27 \\
\hline Localidad = Loc (Is) & 3 & 878,37 & $2,66^{*}$ & 60,59 & $4,13^{*}$ & 34,28 & 0,53 & 180,26 & $4,19^{* *}$ & 145,45 & $2,77^{*}$ \\
\hline Residual & 534 & 330,79 & & 14,66 & & 65,12 & & 43,03 & & 52,41 & \\
\hline
\end{tabular}

gl: Grados de libertad; Cm: Suma de cuadrados medios; F: Valor del estadístico de Fisher Nivel de significación: * $0,01<P<0,05 ; * * P<0,01$

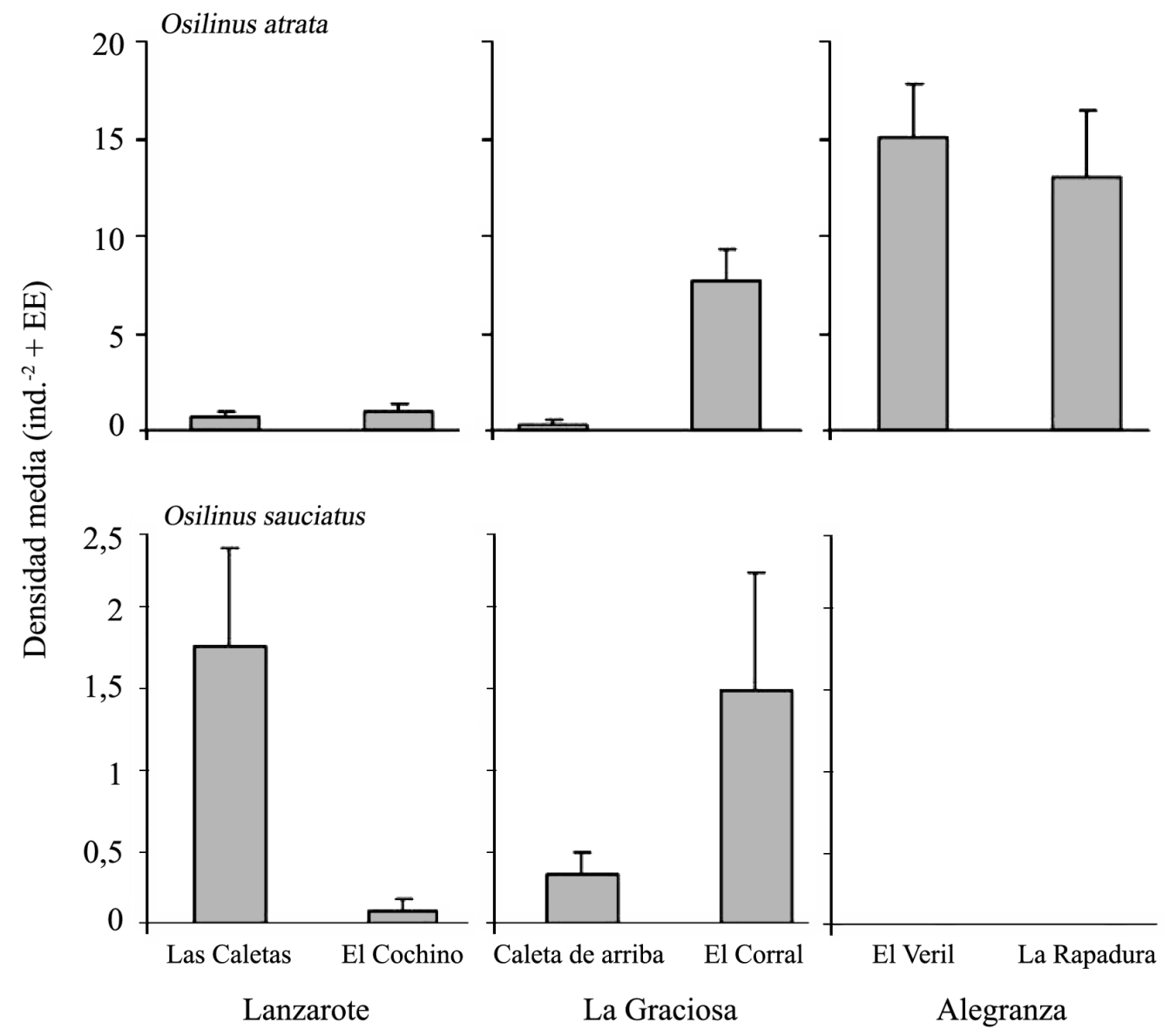

Figura 2

Densidad media por localidad de $\boldsymbol{O}$. atrata y $\boldsymbol{O}$ sauciatus. EE: error estándar

Mean density per location for $O$. atrata and $O$. sauciatus. EE: standard error 
El rango de tallas de $O$. atrata fue de 4-27 $\mathrm{mm}$. La distribución de tallas fue unimodal en la isla de AG, con el rango de tallas 10-12 mm como el más frecuente (Fig. 3). En LG la distribución de tallas también fue unimodal, aunque mostró una frecuencia similar para los rangos de talla 13-15 mm (el más frecuente) y 10-12 mm (Fig. 3). Mientras, la distribución de tallas en L mostró varios rangos de tallas con frecuencias iguales o similares (Fig. $3)$. Se encontraron diferencias significativas para la estructura de talla de $O$. atrata entre islas, y por tanto entre los distintos niveles de influencia humana (Tabla 4). Para $O$. sauciatus, el rango fue de 5-26 mm. En L, la distribución de tallas fue unimodal, con el rango de talla 10-12 mm como el más frecuente. En LG, O. sauciatus

\section{Tabla 4}

Valores del estadístico $\chi^{2}$ para la comparación de las estructura de tallas de cada especie entre islas (= niveles de influencia humana)

Values of the $\chi^{2}$ statistic for the comparison of the size structure of each species among islands (= levels of human influence)

\begin{tabular}{lrrrr}
\hline & g l & AG-LG & \multicolumn{1}{c}{ LG-L } & \multicolumn{1}{c}{ AG-L } \\
\hline O. atrata & 9 & $1752,8^{*}$ & $1006,5^{*}$ & $1023,0^{*}$ \\
O. sauciatus & 9 & $41,0^{*}$ & $13,5 \mathrm{~ns}$ & $41,0^{*}$ \\
P. aspera & 11 & $1946,3^{*}$ & $6,0 \mathrm{~ns}$ & $4007,3^{*}$ \\
P. candei crenata & 11 & $4000,0^{*}$ & $2,0 \mathrm{~ns}$ & $3796,0^{*}$ \\
P. rustica & 11 & $791,5^{*}$ & $33,9^{*}$ & $289,1^{*}$ \\
\hline
\end{tabular}

Nivel de significación: * $P<0,01$ mostró una población estructurada en torno a dos clases de talla, que fueron: 7-9 mm (la más frecuente) y 16-18 mm (Fig. 3). La distribución para ambas islas presentó asimetría positiva, mostrando una cola prolongada en donde se situaron los ejemplares de mayor talla, que fueron los menos abundantes (Fig. 3). Se detectaron diferencias significativas en la estructura de tallas de $O$. sauciatus entre AG y LG y AG y L, pero no entre LG y L (Tabla 4).

\section{Lapas (Patella spp.)}

Se registró un total de 222 ejemplares de $P$. aspera, 179 de $P$. candei crenata y 185 de $P$. rustica, de los cuales un 95, 99 y $74 \%$ se encontraron en AG, respectivamente. Igualmente, un $23 \%$ de los ejemplares de $P$. rustica fue hallado en la isla de L. Se detectaron diferencias significativas entre islas para $P$. aspera, la cual fue más abundante en AG que en LG (prueba SNK, $0,01<P<$ 0,05 ) y L (prueba SNK, $P<0,01$ ) (Tabla 3, Fig. 4); no se registraron diferencias significativas en LG y L. Sin embargo, tanto para $P$. candei crenata (prueba SNK, $P<$ 0,01 ) como para $P$. rustica (prueba SNK, $0,01<P<0,05$ ) se observó una alta variabilidad entre localidades dentro de cada isla, que posiblemente obscureció la detección de diferencias entre islas (Tabla 3; Fig. 4).

El rango de tallas de Patella aspera fue de 8-49 mm, para Patella candei crenata fue de $12-54 \mathrm{~mm}$ y para Patella rustica fue de 3-49 mm. La distribución de tallas de $P$. aspera y $P$. candei crenata no pudo ser definida en L y LG debido a la escasez de ejemplares registrados

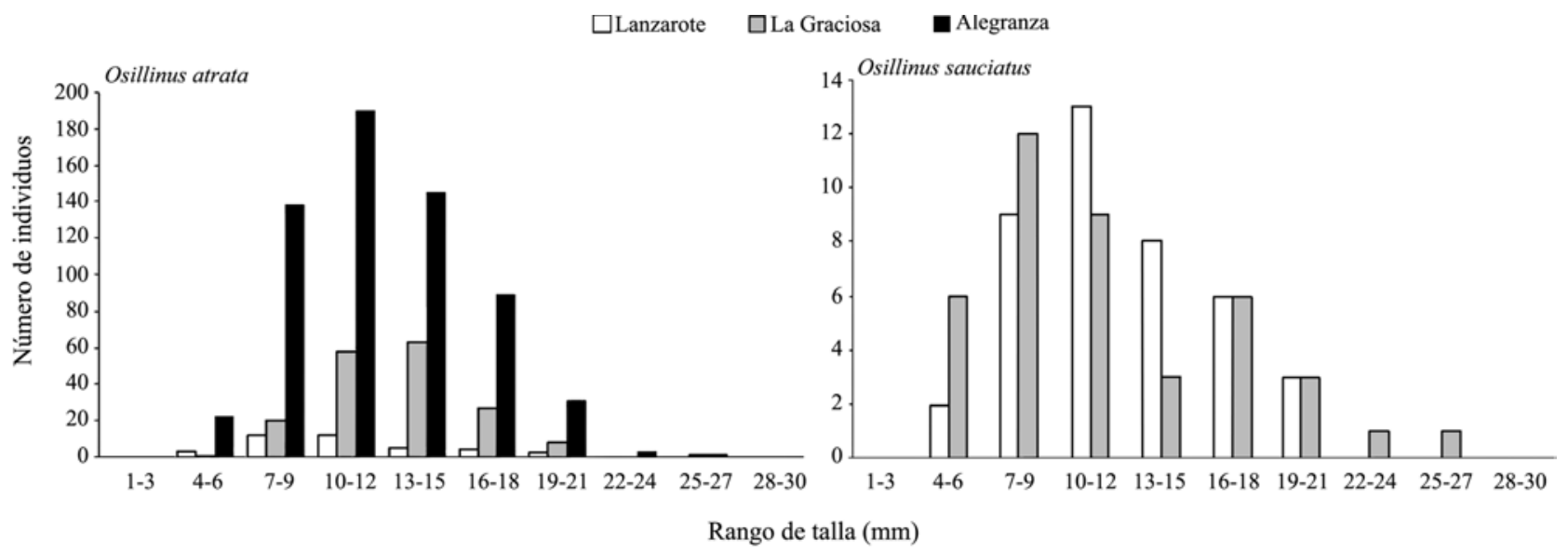

Figura 3

Diagrama de frecuencia de tallas para $O$. atrata y $O$. sauciatus

Size-frequency diagram for $O$. atrata and $O$. sauciatus 


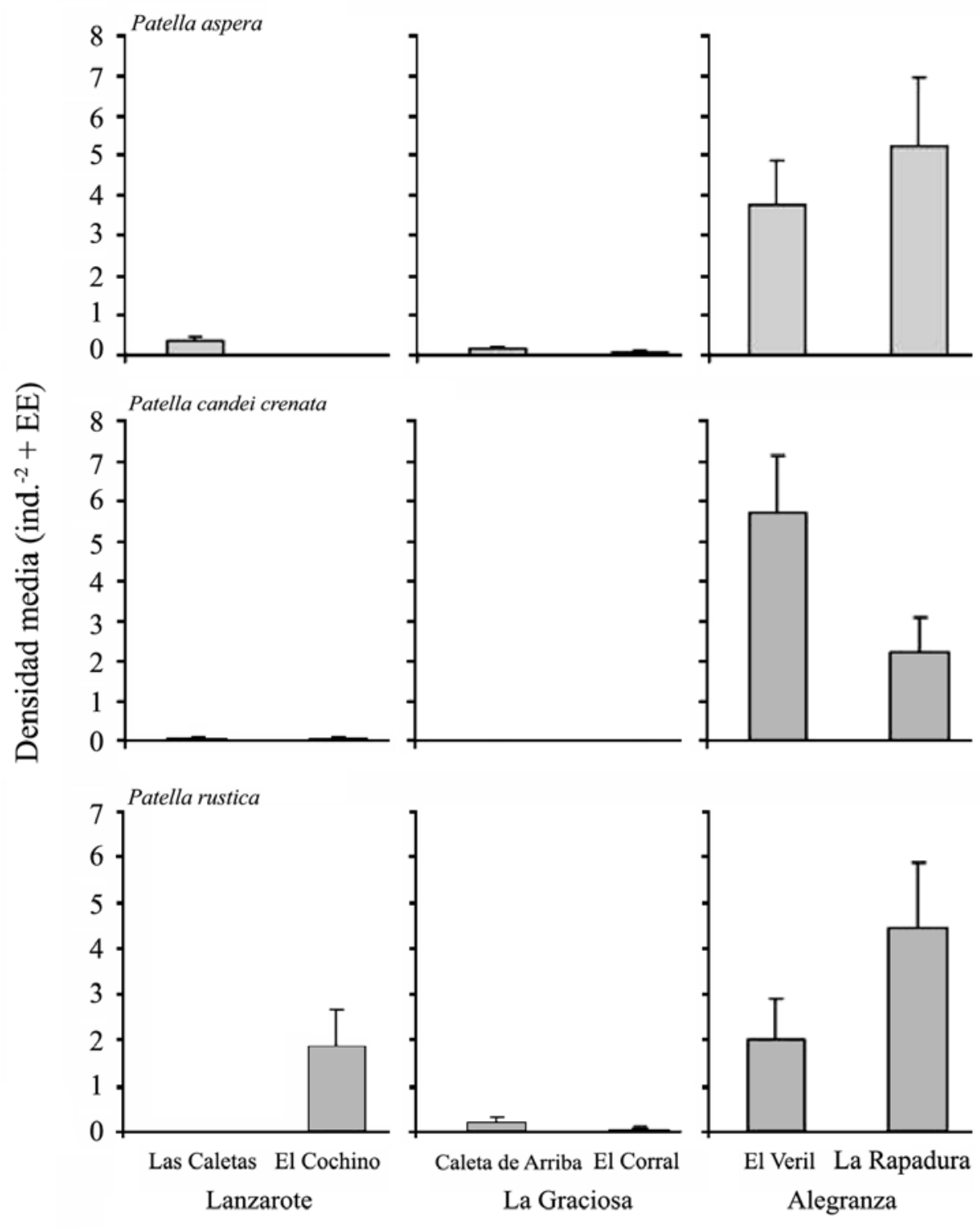

Figura 4

Densidad media por localidad de $P$. aspera, $P$. candei crenata y $P$. rustica. EE: error estándar

Mean density per location for P. aspera, $P$. candei crenata and P. rustica. EE: standard error

(Fig. 5). En AG, en cambio, P. aspera mostró una distribución con varias clases de talla (entre 16 y $35 \mathrm{~mm}$ ) con similares frecuencias y $P$. candei crenata una distribución unimodal, siendo el rango de talla 36-40 mm el más frecuente (Fig. 5). Por otro lado, la distribución de talla de $P$. rustica en L y AG fue unimodal, con el rango de talla 16-20 mm como el más frecuente en ambos casos, y con asimetría positiva (Fig. 5). Los ejemplares registrados en LG no fueron suficientes para definir la distribución de tallas en dicha isla. Se encontraron diferencias significativas para la estructura de tallas de $P$. rustica entre islas; es decir, entre los niveles de influencia humana (Tabla 4). Para P. aspera y P. cande $i$ crenata no se encontraron diferencias significativas entre LG y L, pero sí para el resto de comparaciones (Tabla 4). 


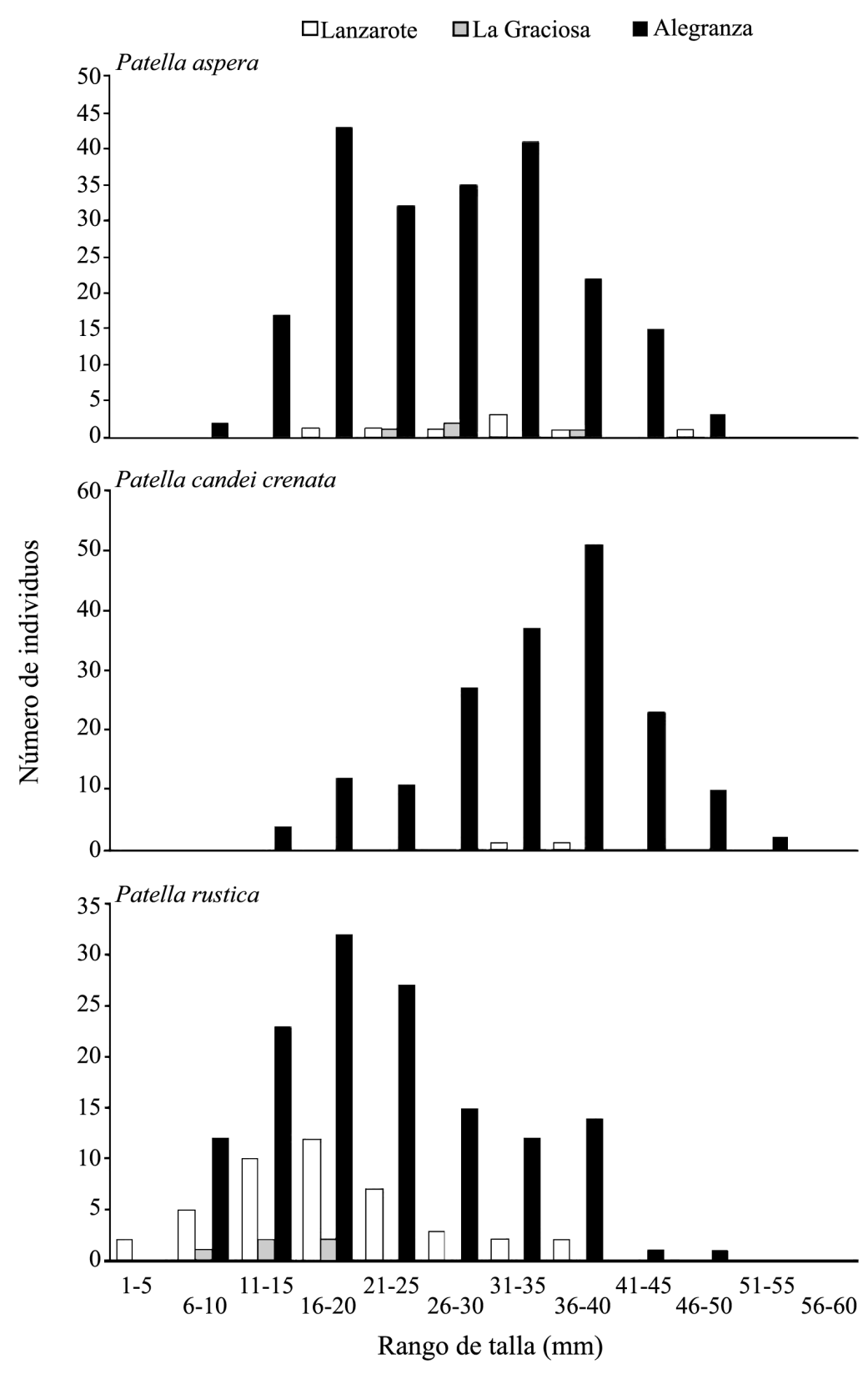

Figura 5

Diagrama de frecuencia de tallas para $P$. aspera, $P$. candei crenata y $P$. rustica

Size-frequency diagram for $P$. aspera, $P$. candei crenata and $P$. rustica

\section{Discusión}

Los resultados de este estudio sugieren que la influencia humana afecta negativamente a las poblaciones de moluscos gasterópodos de interés marisquero en el Archipiélago Canario. Como suponíamos, las especies presentes en áreas sujetas a una influencia humana media $\mathrm{o}$ alta presentaron alteraciones poblacionales respecto a áreas sometidas a menor influencia. Así, y como se ha indicado en diversos estudios (e.g. Hockey \& Bosman 1986, Keough et al. 1993, Lindberg et al. 1998, Roy et al. 2003, Sagarin et al. 2007), la explotación ejercida por el hombre en el Archipiélago Canario ha provocado una disminución de la densidad de las poblaciones y una alteración de la estructura de talla, debido principalmente a la selección de los ejemplares más grandes (Tuya et al. 
2006b). Aunque estadísticamente sólo $O$. atrata y $P$. aspera presentaron diferencias significativas entre las islas (= niveles de influencia humana), la abundancia para cuatro de las cinco especies estudiadas fue mucho mayor en AG (nivel bajo) respecto a LG (nivel medio) y L (nivel alto).

En L, la zona costera está sometida a una alta presión humana, tanto de locales como de turistas, quienes pueden, al mismo tiempo, mariscar y generar una mayor demanda de productos marisqueros. Además, existen numerosas vías de acceso a la costa lo que facilita la actividad de los mariscadores, quienes pueden ahorrar tiempo y energía. Por ejemplo, Adessi (1994) registró para una zona costera de San Diego (California, EUA) que las personas se concentraban cerca de los accesos públicos, lo que resultaba en una mayor perturbación sobre las poblaciones intermareales. No obstante, existen lugares (e.g. varias localidades costeras en California o la localidad denominada 'El Cochino' en este estudio) en los que, a pesar de la lejanía a los accesos públicos, se producen amplias capturas (Sagarin et al. 2007, observación personal). Otros aspectos que facilitan el marisqueo en la zona costera de L son la ausencia de barreras físicas (exceptuando 'El Cochino') y geológicas (e.g. acantilados abruptos) o patrullas de vigilancia. En definitiva, en L se extrae un gran volumen de capturas lo que ha provocado la observación de poblaciones poco densas de burgados y lapas. Aunque no dispongamos de volúmenes de captura para los burgados, nuestras observaciones sobre el terreno indican extracciones tan extensas como las de lapas. En este sentido, se supone que el volumen de extracción es mucho mayor al registrado, debido a que la mayoría de las capturas se realizan ilegalmente; es decir, los mariscadores superan los límites de frecuencia y volúmenes de captura establecidos por las autoridades (BOC, Ley 17/2003, de 10 de abril, de Pesca de Canarias; BOC, Orden de 14 de abril de 2008, por la que se regula temporalmente el marisqueo profesional a pie). El marisqueo ilegal está ampliamente extendido en el Archipiélago Canario, en donde existe una fuerte demanda de productos marisqueros (e.g. lapas), que es aprovechada por los mariscadores para vender sus capturas y obtener así un ingreso extra de recursos económicos.

En el caso de LG, a pesar de estar sometida a una menor influencia humana (nivel medio), el volumen de capturas fue similar a L. Así, las especies de burgados y lapas mostraron igualmente poblaciones poco densas. Para LG se esperaba una menor recolección debido a las limitaciones físicas más extremas (e.g. ausencia de carreteras asfaltadas) o a las restricciones obligatorias sobre el marisqueo (e.g. área de Reserva Marina, patrullas de vigilancia). Sobre este resultado ha podido influir el hecho de que, desde el poblamiento de la isla en el año 1875, las actividades pesqueras - incluyendo el marisqueo- han sido de forma casi exclusiva el medio de subsistencia, ya que la agricultura y la ganadería son casi impracticables debido a las malas condiciones climatológicas y geológicas de la isla (Cabrera 1995, Cabrera \& Cabrera 2004, Leal 2004). Igualmente, a pesar de que en las últimas décadas se ha desarrollado la actividad turística en la isla y ha descendido la actividad pesquera (Cabrera \& Cabrera 2004), la actividad marisquera no ha cesado sino que, posiblemente, ha aumentado al convertirse en un negocio lucrativo; los recursos marisqueros capturados son vendidos en los múltiples establecimientos de restauración tanto de LG como en la isla vecina de $\mathrm{L}$.

Los valores de densidad registrados en AG fueron, en cambio, de 10 a 15 veces superiores a los encontrados en LG y L. Este resultado parece mostrar que la baja influencia humana (casi inexistente) en AG es la causa del buen estado de conservación de las poblaciones de burgados y lapas. La isla de AG está deshabitada, posee extremas restricciones físicas para el acceso (e.g. isla alejada a una distancia > 11 millas náuticas de LG y L, ausencia de puerto y red viaria, restricciones legales sobre el marisqueo (e.g. área de Reserva Marina, patrullas de vigilancia) e incluso es necesario un permiso de la autoridad local (Cabildo de Lanzarote) para el acceso a la isla. En Canarias, existen otros ejemplos de áreas de acceso restringido a la población (e.g. zonas militares), o sobre las cuales se imponen temporalmente estrictas condiciones de vigilancia y prohibiciones de captura (e.g. isla de Fuerteventura), en donde se han encontrado las poblaciones más densas de $P$. aspera (Navarro et al. 2005, Ramírez \& Haroun 2006).

Por otra parte, la ausencia de O. sauciatus en AG en este estudio pudo estar relacionada con su gran variabilidad espacial, que se relaciona con sus preferencias de hábitat y las características de las costas rocosas (Ramírez et al. 2005). Este tróquido parece preferir plataformas rocosas de poca pendiente y gran extensión. En este caso, todas las plataformas analizadas mostraron pendientes similares, pero las situadas en AG fueron de menor longitud que las ubicadas en LG y L, lo que pudo influir en nuestro resultado. El efecto de la variabilidad natural sobre la distribución de $O$. sauciatus parece un caso particular en comparación con el resto de especies, ya que ésta presenta, además, una distribución limitada a determinadas islas del Archipiélago Canario (Ramírez et al. 2005), y no así el resto, las cuales se distribuyen ampliamente por todas las islas (Ramírez et al. 2008). 
Nuestros resultados mostraron diferencias significativas entre las estructura de tallas de todas las especies registradas en AG y aquellas detectadas en LG y L. En estas dos últimas islas, las poblaciones de burgados y lapas no sólo mostraron una desaparición de los ejemplares de mayor talla, como se ha observado de manera general para todo el Archipiélago (Navarro et al. 2005, Ramírez et al. 2005) o en otras áreas geográficas con especies similares (e.g. Keough et al. 1993, Ferraz et al. 2001, Roy et al. 2003, Branch \& Odendaal 2003, Sagarin et al. 2007), sino una disminución de ejemplares para la mayoría de los rangos de talla. Esta observación puede mostrar un fenómeno de sobreexplotación de las poblaciones, el cual puede estar afectando al éxito reproductor de las diferentes especies. Por ejemplo, se sabe que algunas especies (e.g. P. aspera) son hermafroditas proterándricas, y por tanto, la desaparición de los ejemplares adultos (los de mayor tamaño y con mayores gónadas) provocaría grandes desviaciones de un sex ratio ideal 1:1 (Peña 1996, Hawkins et al. 2000, Delany et al. 2002, Branch \& Odendaal 2003). Además, Osilinus spp. y $P$. rustica no poseen poblaciones submareales que sirvan de refugio y que soporten el reclutamiento posterior de las poblaciones situadas en la zona intermareal.

Por otro lado, las diferentes especies analizadas mostraron en AG poblaciones bien estructuradas, con numerosos ejemplares de gran talla. Aunque en algunas ocasiones (principalmente en verano, debido al buen estado climatológico y de la mar), se producen episodios de marisqueo ilegal, éstos no parecen haber sido suficientes para alterar la estructura de talla de las poblaciones; ni siquiera, parecen haber provocado la estructura de talla presentada por $P$. rustica, la cual mostró una estructura de talla desplazada hacia los ejemplares de menor talla. La estructura de talla de esta especie en AG parece ser el patrón natural y no así en LG y L (ver comentarios arriba), en donde es recolectada por los mariscadores para consumo propio cuando no encuentran las otras dos especies (observación personal). En AG también se observó que los ejemplares de menor talla (reclutas) eran escasos o inexistentes para todas las especies. Esto ha sido igualmente registrado para otras especies de lapas (Cantos et al. 1994, Branch \& Odendaal 2003) u otros moluscos gasterópodos (Edgar \& Barret 1999) para zonas sometidas a diferente presión humana, como por ejemplo en áreas marinas protegidas. En esos casos, se sugirió que dicho patrón respondía a dos posibles explicaciones: (1) competencia entre ejemplares grandes y pequeños y (2) incremento de la predación sobre los ejemplares pequeños debido al aumento de predadores dentro del área marina protegida. En nuestro caso, son necesarios más estudios para contrastar tales causas; de hecho, para detectar cambios entre hábitats perturbados y no perturbados por la acción humana se sugieren muestreos regulares en el tiempo (Adessi 1994).

La variabilidad natural (e.g. variabilidad espacial) en la abundancia para las especies de burgados y lapas es notable para todo el Archipiélago Canario (Navarro et al. 2005, Ramírez et al. 2005), así como para otras especies muy relacionadas (e.g. Ramírez et al. 2009). Aún así, dicha variabilidad no parece ser suficiente para explicar las grandes discrepancias registradas entre islas para la abundancia y la estructura de talla en este estudio. Los múltiples impactos producidos por el hombre sobre las poblaciones naturales (principalmente la extracción no regulada, poco selectiva y prolongada en el tiempo), parecen ser las causas más importantes para generar las alteraciones observadas. Por ejemplo, Tuya et al. (2006a) indicaron que la explotación podía enmascarar la regulación producida por procesos 'bottom-up' para las poblaciones intermareales. Igualmente, Ramírez et al. (2008) señalaron que las perturbaciones antrópicas podían influir sobre los patrones de distribución espacial (sobre la abundancia y la talla) del molusco depredador Stramonita haemastoma. Más aún, la extinción del ostrero unicolor (Haematopus meadewaldoi) presente en el área de este estudio hasta mediados del siglo XX (e.g. Moreno 1988, De Juana \& Varela 2000) parece estar relacionada con la actividad humana. Ambos competirían por el mismo alimento (e.g. lapas), pero diferenciándose en que los humanos pueden seleccionar ejemplares de gran talla no accesibles para las aves. En otras áreas geográficas donde existen actualmente ostreros se ha observado: (1) un desplazamiento de los ostreros por los humanos en aquellas zonas frecuentadas por los segundos (Lindberg et al. 1998), y (2) la existencia de lapas de gran talla incluso en donde los ostreros son comunes (Sagarin et al. 2007).

En conclusión, nuestras observaciones plantean serias dudas sobre la efectividad de las regulaciones emanadas de la Ley de Pesca de Canarias y de la Reserva Marina de Interés Pesquero 'Isla de La Graciosa e Islotes al Norte de Lanzarote', dentro de la cual se sitúan LG y AG, debido a la escasa aplicación de las normas sobre el marisqueo y sus medidas de vigilancia. La citada Ley regula, desde el año 2003, las actividades marisqueras en todo el Archipiélago, limitando la frecuencia, el área de desarrollo de la actividad y el volumen y distribución (venta) de las capturas. Además, y dentro de la Reserva Marina de Interés Pesquero, se regula y limita, desde el año 1995, la actividad marisquera. Esta observación concuerda, de cierta forma, con lo apuntado por varios autores, que también han considerado que el estatus legal 
de una zona, como las reservas marinas, son insuficientes cuando no hay una adecuada aplicación de las leyes (e.g. Allison et al. 1998, Boersma \& Parrish 1999, Murray et al. 1999, Sagarin et al. 2007). En reservas marinas con un nivel de vigilancia adecuado se ha observado que sus efectos beneficiosos pueden producirse en cortos períodos de tiempo (1-3 años) desde su declaración (e.g. Durán \& Castilla 1989, Halpern \& Warner 2002). En el presente estudio, este periodo ha sido superado ampliamente (14 años de antigüedad). Por tanto, y a pesar de que no existen datos previos a la creación de este espacio protegido para contrastar las diferencias en abundancia y talla de los recursos estudiados, todo indica a que los bajos valores de abundancia y talla en la isla de LG están fuertemente influenciados por la ineficacia de las medidas de vigilancia dentro de la mencionada Reserva Marina de Interés Pesquero.

\section{Agradecimientos}

Agradecemos al Ministerio de Medio Ambiente, Medio Marino y Rural (Gobierno de España) la financiación parcial de este trabajo. Tres revisores anónimos suministraron comentarios positivos, que ayudaron a mejorar una versión previa del artículo.

\section{Literatura citada}

Addessi L. 1994. Human disturbance and long-term changes on rocky intertidal community. Ecological Applications 4: 786-797.

Allison GW, J Lubchenco \& MH Carr. 1998. Marine reserves are necessary but not sufficient for marine conservation. Ecological Applications 8: 79-92.

Batista C. 2001. El marisqueo en la prehistoria de Gran Canaria. Vector plus 18: 67-76.

Boersma PD \& JK Parrish. 1999. Limiting abuse: marine protected areas, a limited solution. Ecological Economics 31: 287-304.

Branch GM \& F Odendaal. 2003. The effects of marine protected areas on the population dynamics of a South African limpet, Cymbula oculus, relative to the influence of wave action. Biological Conservation 114: 255-269.

Cabrera G. 1995. Del mito del dominio masculino o del matriarcado encubierto: las transformaciones de la familia y su influencia en un contexto insular canario (isla de La Graciosa), pp. 651-656. VI Jornadas de Estudios sobre Lanzarote y Fuerteventura, Cabildo Insular de Lanzarote y Cabildo Insular de Fuerteventura, Arrecife.

Cabrera G \& A Cabrera. 2004. Turismo versus pesca artesanal. A propósito de La Reserva Marina de la Isla de La Graciosa y los Islotes del Norte de Lanzarote. Pasos 2: 1-16.

Cantos R, P Mazón, MD Paredes, ML Picó, E Sales \& P Sánchez-Jerez. 1994. Aportación al conocimiento de las poblaciones de Patella caerulea y $P$. aspera en la reserva marina de Tabarca (Alicante): densidad poblacional y frecuencia de tallas según el tipo de sustrato y grado de presión antrópica. En: Ramos A (ed). Trabajos de campo en la reserva marina de Tabarca, pp. 45-52. Universitat de Alicante-Instituto de Ecología Litoral, Alicante.

Delany J, D McGrath, R O'Riordan \& A Myers. 2002. Reproduction in the intertidal limpets Patella vulgata and Patella ulyssiponensis. En: Myers A (ed). New survey of Clare Island. Marine Intertidal Ecology 3: 91-116. Royal Irish Academy, Dublin.

De Juana E \& JM Varela. 2000. Guía de las aves de España. Península, Baleares y Canarias, 223 pp. Lynx Edicions, Barcelona.

Durán LR \& JC Castilla. 1989. Variation and persistence of the middle rocky intertidal community of central Chile, with and without human harvesting. Marine Biology 103: 555562.

Edgar GJ \& NS Barret. 1999. Effects of the declaration of marine reserves on Tasmanian reef fishes, invertebrates and plants. Journal of Experimental Marine Biology and Ecology 242: 107-144.

Fernández-Palacios JM \& J Martín. 2001. Las islas como experimento de laboratorio. En: Fernández-Palacios JM \& J Martín (eds). Naturaleza de las Islas Canarias. Ecología y conservación, pp. 39-44. Ediciones Turquesa, Santa Cruz de Tenerife.

Ferraz RR, GM Menezes \& RS Santos. 2001. Limpet (Patella spp.) (Mollusca: Gastropoda) exploitation in the Azores, during the period 1993-1998. Arquipélago Suppl. 2(Part B): 59-65.

Guerra-García JM, J Corzo, F Espinosa \& JC GarcíaGómez. 2004. Assessing habitat use of the endangered marine mollusc Patella ferruginea (Gastropoda, Patellidae) in northern Africa: preliminary results and implications for conservation. Biological Conservation 116: 319-326.

Halpern BS \& RR Warner. 2002. Marine reserves have rapid and lasting effects. Ecology Letters 5: 361-366.

Hawkins SJ, HBSM Corte-Real, FG Pannacciulli, LC Weber \& JDD Bishop. 2000. Thoughts on the ecology and evolution of the intertidal biota of the Azores and other Atlantic islands. Hydrobiologia 440: 3-17.

Hayward BW \& FJ Brook. 1981. Exploitation and redistribution of flax snail (Placostylus) by the prehistoric maori. New Zealand Journal of Ecology 4: 33-36.

Hockey PAR \& AL Bosman. 1986. Man as an intertidal predator in Transkei: disturbance, community, convergence and management of a natural food resource. Oikos 46: 3-14.

Keough MJ, GP Quinn \& A King. 1993. Correlations between human collecting and intertidal mollusc populations on rocky shores. Conservation Biology 7: 378-390.

Kido JS \& SN Murray. 2003. Variation in owl limpet Lottia gigantea population structures, growth rates, and gonadal production on southern California rocky shores. Marine Ecology Progress Series 257: 111-124. 
Kingsford MJ, AJ Underwood \& SJ Kennelly. 1991. Humans as predators on rocky reefs in New South Wales, Australia. Marine Ecology Progress Series 72: 1-14.

Leal M. 2004. Lanzarote y Fuerteventura: consideraciones en torno a la actividad pesquera y su incidencia en las hambrunas de antaño. Testimonios de prensa, Tomo I: 231256. VI Jornadas de Estudio sobre Lanzarote y Fuerteventura. Cabildo insular de Lanzarote y Cabildo insular de Fuerteventura, Arrecife.

Lindberg DR, JA Estes \& KI Warheit. 1998. Human influences on trophic cascades along rocky shores. Ecological Applications 8: 880-890.

Mannino MA \& KD Thomas. 2001. Intensive mesolithic exploitation of coastal resources? Evidence from a shell deposit on the Isle of Portland (Southern England) for the impact of human foraging on populations of intertidal rocky shore molluscs. Journal of Archaeological Science 28: 11011114.

Milazzo M \& AA Ramos-Esplá. 2000. Methods for studying the impact of trampling on rocky shallow areas. En: Goñi R, M Harmelin-Vivien, F Badalamenti, L Le Diréach, \& F Bernard (eds). Introduction guide to methods for selected ecological studies in marine reserves, pp. 63-68. GIS Posidonie, Marsella.

Moreno JM. 1988. Guía de las aves de las Islas Canarias, 231 pp. Editorial Interinsular Canaria, Santa Cruz de Tenerife.

Murray SN, TG Denis, JS Kido \& JR Smith. 1999. Human visitation and the frequency and potential effects of collecting on rocky intertidal populations in southern California marine reserves. CalCOFI Report 40: 100-106.

Navarro PG, R Ramírez, F Tuya, C Fernández-Gil, P Sánchez-Jerez \& RJ Haroun. 2005. Hierarchical analysis of spatial distribution patterns of Patellid limpets in the Canary Islands. Journal of Molluscan Studies 71: 67-73.

Núñez J, A Brito \& J Barquín. 1994. Cartografía de la distribución, biología y evaluación de los recursos marisqueros de moluscos litorales (lapas, oreja de mar y mejillón), primera fase: La Palma, Tenerife, Gran Canaria y Fuerteventura, 321 pp. Departamento de Biología Animal (Universidad de La Laguna) y Consejería de Agricultura, Pesca y Alimentación (Gobierno de Canarias), La Laguna.

Núñez J, MC Brito, R Riera, JR Docoito \& O Monterroso. 2003. Distribución actual de las poblaciones de Patella candei D'Orbigny, 1840 (Mollusca, Gastropoda) en las Islas Canarias. Una especie en peligro de extinción. Boletín del Instituto Español de Oceanografía 19: 371-377.

Peña J. 1996. Estudio de los ciclos reproductores de cuatro especies de lapas y dos tróquidos (Gastropoda, Prosobranchia) del piso intermareal de la costa vasca. Tesis doctoral, Universidad Autónoma de Madrid, Madrid, 441 pp.
Ramírez R \& RJ Haroun. 2006. Estado de las poblaciones de diversos moluscos presentes en las costas de la isla de Fuerteventura, 20 pp. BIOGES, Universidad de Las Palmas de Gran Canaria, Las Palmas de Gran Canaria.

Ramírez R, F Tuya \& RJ Haroun. 2008. El intermareal canario. Poblaciones de lapas, burgados y cañadillas, 54 pp. BIOGES, Universidad de Las Palmas de Gran Canaria, Las Palmas de Gran Canaria.

Ramírez R, F Tuya \& RJ Haroun. 2009. Spatial patterns in the population structure of the whelk Stramonita haemastoma (Linnaeus, 1766) (Gastropoda: Muricidae) in the Canarian Archipelago (eastern Atlantic). Scientia Marina 73: 431-437.

Ramírez R, F Tuya, P Sánchez-Jerez, C Fernández-Gil, O Bergasa, RJ Haroun \& JJ Hernández-Brito. 2005. Population structure and spatial distribution of the gastropod molluscs Osilinus atrata and Osilinus sauciatus in the rocky intertidal zone of the Canary Islands. Ciencias Marinas 31: 697-706.

Roy K, AG Collins, BJ Becker, E Begovic \& JM Engle. 2003 Anthropogenic impacts and historical decline in body size of rocky intertidal gastropods in southern California. Ecology Letters 6: 205-211.

Sagarin RD, RF Ambrose, BJ Becker, JM Engle, J Kido, SF Lee, CM Miner, SN Murray, PT Raimondi, D Richards \& C Roe. 2007. Ecological impacts on the limpet Lottia gigantea populations: human pressure over a broad scale on island and mainland intertidal zones. Marine Biology 150: 399-413.

Stephenson TA \& Stephenson A. 1949. The universal features of zonation between tide-marks on rocky coasts. The Journal of Ecology 37: 289-305.

Thompson RC, TP Crowe \& SJ Hawkins. 2002. Rocky intertidal communities: past environmental changes, present status and predictions for the next 25 years. Environmental Conservation 29: 168-191.

Tuya F, C García-Díez, F Espino \& RJ Haroun. 2006b. Assessment of the effectiveness of two marine reserves in the Canary Islands (eastern Atlantic). Ciencias Marinas 32: 505-522.

Tuya F, R Ramírez, P Sánchez-Jerez, RJ Haroun, AJ González-Ramos \& J Coca. 2006a. Coastal resources exploitation can mask bottom-up mesoscale regulation of intertidal populations. Hydrobiologia 553: 337-344

Underwood AJ. 1997. Experiments in ecology: their logical design and interpretation using analysis of variance, 504 pp. Cambridge University Press, Cambridge. 